\title{
Fractura subsecuente y reingreso hospitalario relacionado a fracturas por fragilidad en Unidad de Coordinación de Fracturas de Ortogeriatría
}

\author{
Subsequent fracture and hospital re-entry related to fragility fractures \\ in Orthogeriatrics unit of Fracture Liaison Services
}

\author{
Wendy Alejandra Reyes-Guerrero, * Evelyn Alina Guillermo-Nuncio, ${ }^{*}$ Kevin Cristian Ramírez-Salas, \\ Karen Sofía Aguilera-De Alba, ${ }^{\ddagger}$ Paola Daniela Zapata-Reyes, ${ }^{\ddagger}$ Saraí Lizbeth Baldenebro-Lugo, $\$$ \\ Hugo Rolando Nieto-Sandoval, * Juan Carlos Viveros-Garcíall \\ *Servicio de Traumatología y Ortopedia; ¥Médico Pasante de Servicio Social; § Jefe de Investigación; \\ Médico Internista y Geriatra. Servicio de Medicina Interna. \\ "Hospital Regional ISSSTE León. México.
}

\begin{abstract}
Resumen
Introducción: Las fracturas por fragilidad tienen una alta tasa de complicaciones, costos y son generadores de dependencia. Los modelos de prevención secundaria de fracturas han demostrado disminuir la tasa de refractura y ser costo-efectivos. Objetivo: Conocer la tasa de refractura y reingreso hospitalario tras una fractura por fragilidad como primera fase del proyecto de FLS o unidad de coordinación de fracturas. Material y métodos: Estudio retrospectivo, transversal, descriptivo. Se incluyeron las fracturas por fragilidad ingresadas del 01 de enero de 2017 al 31 de diciembre de 2019 en el Hospital Regional ISSSTE León, México. Resultados: En el periodo descrito hubo un total de 343 fracturas por fragilidad, con edad media de 69 años y $79 \%$ de pacientes fueron del género femenino. La fractura más frecuente fue la de cadera (33.8\%) seguida de la fractura de radio distal $(25.1 \%)$. Sólo en $8.5 \%$ de los pacientes se menciona el diagnóstico de osteoporosis. La mayoría de los pacientes recibieron tratamiento quirúrgico (86.6\%), $40.5 \%$ de los pacientes reingresaron en los siguientes 12 meses por lo menos una vez y 17 pacientes tuvieron una nueva fractura por fragilidad (12\%). Discusión: La tasa de refractura 0 fractura subsecuente de nuestro hospital al primer año posterior a una fractura es alta en comparación con otros centros. Conclusiones: Se demuestra que la tasa de refracturas y reingresos en nuestro hospital se encuentra por encima de la tasa esperada en hospitales con una FLS. La creación de este modelo es prioridad, ya que existe evidencia de que su implementación mejora la calidad asistencial.
\end{abstract}

Palabras clave: Fracturas por fragilidad, ortogeriatría, unidad de coordinación de fracturas.

\section{Abstract}

Introduction: Fragility fractures have a high rate of complications, costs and are dependency generators. Secondary fracture prevention models have been shown to decrease the refracture rate and to be cost effective. Objective: To know the rate of refracture and hospital readmission after a fragility fracture as the first phase of the FLS project or Fracture Coordination Unit. Material and methods: Retrospective, cross-sectional, descriptive study. Fragility fractures admitted from January 1, 2017 to December 31, 2019 at Hospital Regional ISSSTE León, Mexico were included. Results: In the period described, a total of 343 fragility fractures, with a mean age of 69 years, $79 \%$ of patients were female. The most frequent fracture was the hip fracture (33.8\%) followed by the distal radius (25.1\%). Only in $8.5 \%$ of patients is the diagnosis of osteoporosis mentioned. Most of the patients received surgical treatment (86.6\%). $40.5 \%$ of the patients were readmitted in the following 12 months at least once. 17 patients had a new fragility fracture (12\%). Discussion: The rate of refracture or subsequent fracture in our hospital in the first year after a fracture is high compared to other centers. Conclusions: It is shown that the rate of refractures and readmissions in our hospital is above the rate expected in hospitals with an FLS. The creation of this model is a priority since there is evidence that its implementation improves the quality of care.

Keywords: Fragility fractures, orthogeriatrics, Fracture Liaison Services.

Correspondencia:

Wendy Alejandra Reyes-Guerrero

E-mail: alejandrawendy@hotmail.com

Recibido: 19-11-2021. Aceptado: 19-12-2021.
Citar como: Reyes-Guerrero WA, Guillermo-Nuncio EA, RamírezSalas KC, Aguilera-De Alba KS, Zapata-Reyes PD, Baldenebro-Lugo $S L$ et al. Fractura subsecuente y reingreso hospitalario relacionado a fracturas por fragilidad en Unidad de Coordinación de Fracturas de Ortogeriatría. Orthotips. 2022; 18 (1): 29-32. https://dx.doi. org/10.35366/103729 


\section{Introducción}

Las fracturas por fragilidad tienen una alta tasa de complicaciones, costos y son generadores de dependencia. Los modelos de prevención secundaria de fracturas han demostrado disminuir la tasa de refractura o fractura subsecuente y ser costo-efectivos. ${ }^{1}$

Las llamadas fracturas por insuficiencia, o en ocasiones fracturas osteoporóticas, corresponden a la solución de continuidad ósea que generalmente ocurre después de un trauma de baja energía con mecanismo de bajo impacto. ${ }^{2}$ La Organización Mundial de la Salud (OMS) reconoce la fragilidad ósea como aquélla donde existe un trastorno esquelético caracterizado por baja masa ósea y/o deterioro de la microarquitectura del tejido asociado a aumento de riesgo de fractura. ${ }^{3}$ La osteoporosis es la enfermedad sistémica con densidad mineral ósea (DMO) medible por densitometría ósea inferior a -2.5 DE (desviaciones estándar) en comparación con la media de adultos jóvenes (T-score). ${ }^{4}$ El riesgo de fractura subsecuente fue más alto para los que presentaban osteoporosis; sin embargo, aproximadamente de 5 a $23 \%$ de las refracturas y la mortalidad podrían atribuirse a las fracturas iniciales con un nivel de DMO normal. ${ }^{5}$

También existe un subdiagnóstico de fractura por fragilidad asociada a osteoporosis, ya que a $90 \%$ de los pacientes hospitalizados, afectados por fractura de cadera, no se les diagnostica a pesar de que $6 \%$ tenían antecedente de fractura previa y que de 2.9 a $5 \%$ de estos pacientes nuevamente presentaron fractura posterior al primer evento, tanto en localización de cadera u otros sitios, ignorándose el diagnóstico de osteoporosis y tratando la fractura aisladamente. ${ }^{6}$

La unidad de ortogeriatría es un modelo multidisciplinario para disminuir la estancia media hospitalaria, la mortalidad y las complicaciones postoperatorias; permite la recuperación de la funcionalidad previa, disminuye el grado de dependencia y costos asociados. ${ }^{7}$

Proporciona un enfoque holístico para identificar a los pacientes y las intervenciones basadas en evidencia con el fin de prevenir fracturas posteriores. El principal desafío al que se enfrentan los sistemas de salud en la actualidad es garantizar que las mejores prácticas se brinden de manera efectiva en el entorno de atención médica local. 8,9

Se ha reportado internacionalmente una tasa de fractura subsecuente de $7.7 \%$ posterior a la implementación del modelo FLS comparado con la tasa de $10.9 \%$ previo a su establecimiento. ${ }^{10}$ En algunos estudios disminuye hasta $5 \%$ la presentación de fractura subsecuente en un periodo de cinco años después de presentar la fractura inicial. ${ }^{11}$

Una fractura previa es un fuerte predictor de fractura posterior; sin embargo, las tasas de tratamiento postfractura son bajas. ${ }^{12}$ Otros factores descritos son país de residencia, edad, sexo, familia, antecedentes, uso de corticosteroides, tabaquismo y antecedentes de fractura por fragilidad. ${ }^{13}$

Las FLS fueron costo-efectivas en comparación con la atención habitual, independientemente de la intensidad del programa o del país en el que se implementó el proyecto. ${ }^{14}$

Actualmente, a menos de un tercio de las mujeres postmenopáusicas y en menor cantidad a hombres del mismo grupo etario se les prescriben tratamientos activos para reducir el riesgo de fracturas. ${ }^{15}$ Por lo que una unidad de coordinación de fracturas es relevante en el manejo de población vulnerable tomando en consideración la cultura de prevención, en este caso de futuras fracturas.

\section{Material y métodos}

Objetivos. Conocer la tasa de fractura subsecuente y reingreso hospitalario tras una fractura por fragilidad al comienzo del proyecto de unidad de coordinación de fracturas de ortogeriatría o FLS en un hospital de tercer nivel.

Caracterización de fracturas por fragilidad para implementar parámetros de calidad en atención.

Métodos. En un estudio retrospectivo, transversal y descriptivo se incluyeron pacientes con fractura por fragilidad del 01 de enero de 2017 al 31 de diciembre de 2019 en el Hospital Regional ISSSTE León, México.

Se realizó la revisión de expedientes físicos con el programa SPSS versión 20 para el análisis estadístico básico. Se obtuvo promedio de edad, porcentaje para variables cualitativas y media para variables continuas.

\section{Resultados}

En el periodo descrito hubo un total de 343 fracturas por fragilidad, con edad media de 69 años y $79 \%$ de los pacientes fueron del género femenino. La fractura más frecuente fue la de cadera (33.8\%) seguida de la fractura de radio distal tipo Colles (25.1\%). Sólo en $8.5 \%$ de los pacientes se menciona el diagnóstico de osteoporosis. La mayoría de los pacientes recibieron tratamiento quirúrgico (86.6\%). De los pacientes, 
40.5\% reingresaron en los siguientes 12 meses por lo menos una vez y 17 pacientes tuvieron una nueva fractura por fragilidad (12\%) (Tabla 1).

\section{Discusión}

Se demuestra mediante este trabajo que la tasa de refractura en nuestro hospital al primer año posterior a una fractura es alta en comparación con otros centros. En un metaanálisis de 2018 por Wu CH y colaboradores fue de $10.9 \%$, mientras que en el Hospital Regional ISSSTE de León, Guanajuato, México fue de $12 \%$. Este estudio también arroja un porcentaje significativo de reingreso a hospital de $40.5 \%$, lo que se asocia a una elevación de los costos en salud, por lo que buscamos que la correcta implementación a lo largo del tiempo de una FLS contribuya a disminuir estos parámetros poco alentadores.

Otro aspecto que considerar es la dependencia que causa una fractura subsecuente, con costos no valorados extrahospitalarios que afectan la dinámica social de un individuo.

La edad en promedio fue de $69 \pm 13$ años, y el sexo femenino concuerda con la literatura internacional. El porcentaje de pacientes con diagnóstico previo de osteoporosis fue de $8.5 \%$, lo que se acerca al $90 \%$ descrito de pacientes sin diagnóstico.

Tabla 1: Características descriptivas de fracturas por fragilidad en el periodo 2017-2019 ( $\mathrm{N}=343$ ).

\begin{tabular}{lc}
\hline Variable & $n(\%)$ \\
\hline Edad (años) & \\
Género femenino & $69.2 \pm 13^{*}$ \\
Fractura índice & $271(79.0)$ \\
$\quad$ Vertebral & \\
$\quad$ Cadera & $0(0)$ \\
$\quad$ Colles & $116(33.8)$ \\
$\quad$ Miembro superior & $86(25.1)$ \\
$\quad$ Miembro inferior & $43(12.5)$ \\
$\quad$ Fémur & $88(25.7)$ \\
Osteoporosis diagnosticada al ingreso & $10(2.9)$ \\
Quirúrgico & $29(8.5)$ \\
Mortalidad intrahospitalaria & $297(86.6)$ \\
Reingreso a 12 meses & $3(0.9)$ \\
Número de reingresos & $139(40.5)$ \\
Refractura por fragilidad & $1(1-2)^{\star *}$ \\
Neumonía & $17(12.0)$ \\
Reintervención ortopédica & $12(8.5)$ \\
\hline
\end{tabular}

* Media \pm desviación estándar.

** Mediana (rango intercuartil).
En cuanto al tratamiento quirúrgico de fractura por fragilidad, en nuestro hospital lo ameritaron 297 (86.6\%) pacientes, lo cual se asocia a mayor probabilidad de complicaciones por estancia intrahospitalaria, como la neumonía que presentó $8.5 \%$ de los pacientes.

Todo lo anterior hace evidente que en nuestro medio es de gran importancia establecer los factores asociados de refractura y reingreso por fracturas por fragilidad, las cuales pueden prevenirse con un adecuado conocimiento del manejo óptimo de la población vulnerable. Se estima un aumento de la población geriátrica en los próximos 20 años, por lo que tomará mayor relevancia la implementación de las FLS para un tratamiento coordinado con especialistas en el área de traumatología y ortopedia con ortogeriatría.

\section{Conclusiones}

La tasa de fractura subsecuente o refractura de $12 \%$ en nuestro hospital se encuentra por encima de la tasa esperada en hospitales con una FLS (10.9\%). La creación de este modelo es prioridad, ya que existe evidencia de que su implementación mejora la calidad asistencial y disminuye hasta $5 \%$ la tasa de fracturas subsecuentes, lo que conduce a una baja en costos de tratamiento a largo plazo. En la actualidad, con el aumento de la población mundial de adultos mayores, la proporción de pacientes que reciben medidas eficientes de prevención de fracturas en este grupo etario es alarmantemente baja, siendo un problema de salud pública.

Las fracturas por fragilidad se han descrito en la literatura internacional asociadas al género femenino, en pacientes mayores de 65 años, siendo la fractura de cadera la de mayor número de incidencias, lo que es similar a lo encontrado en este trabajo. Partiendo de este conocimiento, se esperaría que en un futuro los profesionales de la salud que detecten estas variables en los pacientes susceptibles, los canalicen a una unidad de coordinación de fracturas (FLS) para una temprana valoración.

\section{Referencias}

1. Mackey PA, Rosenthal LD, Mi L, Whitaker MD. Subsequent fracture prevention in patients 50 years and older with fragility fractures: A quality improvement initiative: A quality improvement initiative. J Healthc Qual. 2019; 41 (1): 17-22.

2. Sanchez-Riera $L$, Wilson N. Fragility fractures \& their impact on older people. Best Pract Res Clin Rheumatol. 2017; 31 (2): 169-191. 
3. World Health Organization. Assessment of fracture risk and its application to screening for postmenopausal osteoporosis: report of a WHO study group ["meeting held in Rome from 22 to 25 June 1992]: World Health Organization; 1994.

4. Compston J, Bowring C, Cooper A, Cooper C, Davies C, Francis $\mathrm{R}$, et al. Diagnosis and management of osteoporosis in postmenopausal women and older men in the UK: National Osteoporosis Guideline Group (NOGG) update 2013. Maturitas. 2013; 75 (4): 392-396.

5. Bliuc D, Alarkawi D, Nguyen TV, Eisman JA, Center JR Risk of subsequent fractures and mortality in elderly women and men with fragility fractures with and without osteoporotic bone density: the Dubbo Osteoporosis Epidemiology Study: Subsequent fracture and mortality risk according to bone density levels. J Bone Miner Res. 2015; 30 (4): 637-646.

6. Leyton Pavez CE, Devetak Álvarez AR, Paul Espinoza IR. Riesgo de fractura osteoporótica y factores de riesgo asociados en mujeres postmenopáusicas en atención primaria de salud. Rev Costarric Salud Pública. 2018; 27 (1): 3-15.

7. Duarte-Flores J. Las unidades de ortogeriatría en México son una prioridad. Rev Med Inst Mex Seguro Soc. 2018; 56 (4): 332-333.

8. Noordin S, Allana S, Masri BA. Establishing a hospital based fracture liaison service to prevent secondary insufficiency fractures. Int J Surg. 2018; 54 (Pt B):328-332.

9. Mitchell PJ, Cooper C, Fujita M, Halbout P, Akesson K, Costa $M$, et al. Quality Improvement Initiatives in Fragility Fracture Care and Prevention. Curr Osteoporos Rep. 2019; 17 (6): 510-520.
10. Li N, Hiligsmann M, Boonen A, van Oostwaard MM, de Bot RTAL, Wyers CE, et al. The impact of fracture liaison services on subsequent fractures and mortality: a systematic literature review and meta-analysis. Osteoporos Int. 2021; 32 (8): 1517-1530.

11. Wu CH, Tu ST, Chang YF, Chan DC, Chien JT, Lin CH, et al. Fracture liaison services improve outcomes of patients with osteoporosis-related fractures: A systematic literature review and meta-analysis. Bone. 2018; 111: 92-100.

12. Balasubramanian A, Zhang J, Chen L, Wenkert D, Daigle SG, Grauer A, et al. Risk of subsequent fracture after prior fracture among older women. Osteoporos Int. 2019; 30 (1): 79-92.

13. Dang DY, Zetumer S, Zhang AL. Recurrent fragility fractures: a cross-sectional analysis. J Am Acad Orthop Surg. 2019; 27 (2): e85-e91.

14. Wu CH, Kao I, Hung W, Lin S, Liu H, Hsieh M, et al. Economic impact and cost-effectiveness of fracture liaison services: a systematic review of the literature. Osteoporos Int. 2018; 29 (6): 1227-1242.

15. Van Geel T, Bliuc D, Geusens P, Jacqueline R, Dinant G, Tran $T$, et al. Reduced mortality and subsequent fracture risk associated with oral bisphosphonate recommendation in a fracture liaison service setting: a prospective cohort study. PLoS One. 2018; 13 (6): e0198006.

\section{Conflicto de intereses}

Los autores declaran no tener ningún conflicto de intereses. 